\title{
Genetic Modulation Therapy Through Stem Cell Transplantation for Human Immunodeficiency Virus 1 Infection
}

\author{
Varshil Mehta $^{1}$, Divya Chandramohan ${ }^{2}$, Shivika Agarwal ${ }^{3}$ \\ 1. Department of Internal Medicine, Seven Hills Hospital, Mumbai 2. Department of Internal Medicine, \\ UAB Montgomery, Montgomery, USA 3. Internal Medicine, Faridabad, India, faridabad, IND
}

$\square$ Corresponding author: Varshil Mehta, varshil91@gmail.com

Disclosures can be found in Additional Information at the end of the article

\section{Abstract}

Highly active anti-retroviral treatment has changed the dimensions of the outcomes for patients suffering from human immunodeficiency virus (HIV)/acquired immunodeficiency syndrome (AIDS). However, HIV infection is still an ailment which is spreading throughout the world extensively. Given the confinements of the present restorative methodologies and the non-availability of any strategic vaccination against HIV, there is a squeezing need to build a therapeutic treatment.

Viral tropism for HIV includes CD4+ cells, macrophages, and microglial cells, and it is through binding with co-receptors $\mathrm{C}-\mathrm{C}$ chemokine receptor type 5 (CCR5) and $\mathrm{C}$-X-C chemokine receptor type 4 (CXCR4). While these cell types are present in all individuals, there are rare cases that stayed uninfected even after getting exposed to an overwhelming load of HIV. Research revealed a homozygous 32-base pair deletion $(\Delta 32 / \Delta 32)$ in CCR5. After careful consideration, a hypothesis was proposed a few years back that a cure for HIV disease is possible, through hematopoietic stem cells transplantation from a donor homozygous for the CCR5- $\triangle 32$ deletion.

Hematopoietic stem cell (HSC) based quality treatment may serve as a promising tool as these perpetual, self-renewing progenitor cells could be modified to oppose HIV infection. If done properly, the changed HSCs would offer the permanent creation of genetically modified cells that are resistant to HIV infection and/or have improved hostility to viral action which will eventually clear the contaminated cells.

The purpose of this review is to concentrate on two facets of HSC genetic treatment for potentially life-threatening HIV infection: building HIV-resistant cells and designing cells that can target HIV disease. These two strategic approaches can be the frontline of a quality

Received 02/21/2017

Review began 03/03/2017 Review ended 03/05/2017 Published 03/13/2017

\section{(c) Copyright 2017}

Mehta et al. This is an open access article distributed under the terms of the Creative Commons Attribution License CC-BY 3.0., which permits unrestricted use, distribution, and reproduction in any medium, provided the original author and source are credited. treatment plan against HIV infection and, as an individual treatment or a combination thereof, has been proposed to possibly destroy HIV altogether.

Categories: Genetics, HIV/AIDS, Infectious Disease

Keywords: hiv treatment, ccr5 resistance, genetic modulation, hematopoietic stem cell transplantation

\section{Introduction And Background}

As indicated by the Joint United Nations Programme on Human Immunodeficiency Virus/Acquired Immune Deficiency Syndrome (UNAIDS), there were roughly 36.7 million 
individuals living with HIV/AIDS towards the end of 2015. Of these, 1.8 million were children (<15 years old). An alarming 2.1 million people were recently contaminated with HIV in 2015. This encompassed 150,000 < 15-year-olds. The greater part of these children live in subSaharan Africa and were contaminated by their HIV-positive mothers amidst pregnancy, labor, or breastfeeding [1]. Although there has been an increase in awareness regarding HIV infection, people remain uninformed about the same in many parts of the world, especially in the slums of developing countries [2-3].

Despite the success of HIV therapy through the combined antiretroviral treatment delivered, HIV infection continues to be a resilient ailment which keeps spreading rapidly around the world. Highly active antiretroviral treatment (HARRT) has been proven to be of maximum benefit for those individuals who have not yet succumbed to an advanced stage of disease, provided it is started earlier in the disease process and is adhered to properly. In such cases, there is a notable decrease in the plasma HIV viral load to low or even imperceptible levels in many patients. This has changed what generally was conceived as a lethal ailment to a constant infirmity.

Notwithstanding this achievement, antiretroviral treatment is not yet completely impeccable. Chronic inflammation and immune dysfunction frequently result post-usage, and subsequent emerging proof demonstrates that there is enigmatic viral replication in scattered lymphoid organs while the therapy is ongoing [4]. Alongside the other harmful effects of antiretroviral medications, this adds to the increasing detriment of non-AIDS morbidity and mortality [5-6].

The HAART regimen requires strict adherence to therapy and numerous patients are noncompliant. Additionally, in asset-restricted nations, access to treatment is capricious and seldom uninterrupted. Given the shortcomings of the present treatment protocol and the nonavailability of a vaccination against HIV, it is imperative to build a therapeutic regimen with a steady rate of success.

A large portion of the general population is susceptible to HIV infection. However, uncommon cases have been noted who stayed uninfected even after getting exposed to an overwhelming load of HIV. Studying these cases have since revealed a homozygous 32 -bp deletion $(\Delta 32 / \Delta 32)$ found in $\mathrm{C}-\mathrm{C}$ chemokine receptor type 5 (CCR5) [7]. While the CD4 T-cell function remains intact, the deletion rendered cells resistant to HIV attachment, since the ordinarily transmitted $\mathrm{R} 5$ viruses utilize the CCR5 receptor together with CD4 to gain entry into cells. This awareness prompted the advancement and utilization of a new class of entry inhibitors that bar CCR5 collaboration with the HIV envelope; this a proposed plausible cure of HIV disease, accomplished through an allogeneic stem cell transplantation from a donor homozygous for the CCR5- $\triangle 32$ deletion [8].

Consequently, hematopoietic stem cell (HSC) based quality treatments have developed as a promising tool, as these cells could be modified and tuned to oppose HIV infection [9-10]. If they are effectively engrafted, the changed HSCs could offer consistent, long-haul creation of genetically modified cells that are resistant to HIV infection and/or have improved hostility to viral action to clear contaminated cells. If the host is successfully repopulated with an HIVresistant hematopoietic framework to not provide attachment sites even given a heavy viral load, then a lasting cure can be accomplished.

This review concentrates on the factors of HSC genetic treatments which potentially help in treating/preventing life-threatening HIV infection by building HIV-resistant cells and making cells which could target the virus. These two approaches could replace the frontline treatment against HIV infection and can possibly help in eliminating HIV altogether. 


\section{Cureus}

\section{Review}

\section{Co-receptors for the passage of HIV into CD4+}

Viral replication requires cellular gene expression processes and activated CD4+ cells which are the primary targets of replicative HIV infection. Thus, HIV disease leads to a decrease in activated memory CD4+ T-cells, the greater part of which exist in the gastrointestinal (GI) mucosa [11-13]. The HIV viral access into CD4+ cells requires proper interaction with a cell surface co-receptor - for the most part, either CCR5 or CXCR4 [8]. The picture is depicted below in Figure 1 [14].

CD4 Attachment

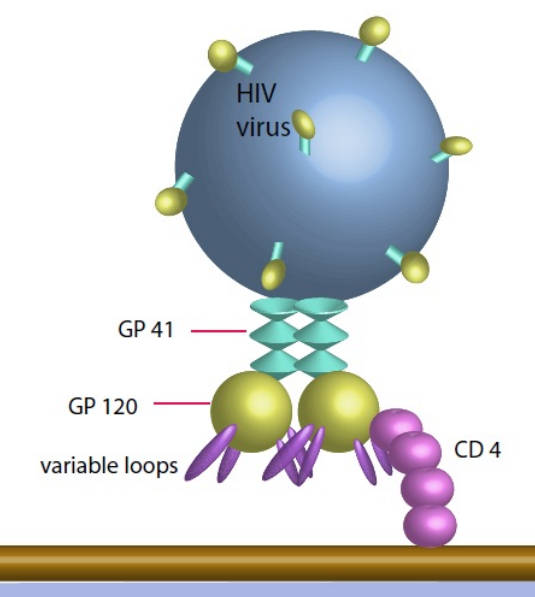

CELL
Co-receptor attachment

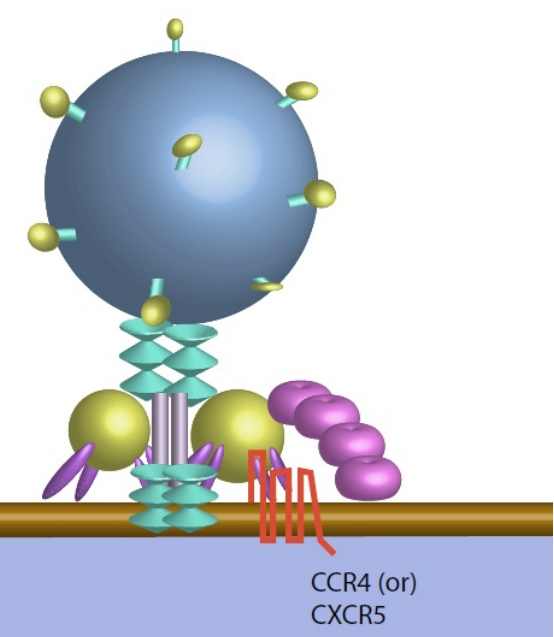

\section{FIGURE 1: HIV attachment process}

Adapted from US National Institutes of Health - National Institute of Allergy and Infectious Diseases [14].

\section{CCR5 co-receptor}

Out of the two, CCR5 is more basic as a co-receptor, and CCR 5 tropism represents a larger share of transmittable HIV-1 strains [15]. The chemokine CCR5 is a vital component in the immune system, where it is profoundly expressed on macrophages and CD4 T-cells [16]. The chromosome 3 in the p21.3-p24 zone expresses the gene for CCR5. In the coding area of CCR5, there are known to be no less than 23 generally uncommon alleles [17]. As expressed, it has been ascertained that people who are homozygous for a deletion of 32 base sets of the CCR5 gene $(\Delta 32)$ are to a great extent resistant to HIV infection [7]. Once HIV-infected, people with a solitary duplicate of the $\Delta 32$ mutation additionally have slower illness progression [18]. Homozygosity of CCR $5-\Delta 32$ in Caucasians was found to be around $1 \%$, with heterozygosity being anything up to $20 \%$ [19]. The recognizable proof of these subjects has made CCR5 an alluring option against antiviral remedial measures. For instance, the Maraviroc, a negative allosteric modulator of the CCR5 receptor, was developed, and it emerged as a fruitful entry inhibitor [20]. Be that as it may, antiviral resistance can still evolve with this medication, and a more lasting procedure focusing on CCR5 would be a more compelling methodology. 


\section{Cureus}

\section{CXCR4 co-receptor}

C-X-C chemokine receptor type 4 (CXCR4) are associated with various physiological processes, for example, B-cell and T-cell development and also as part of cardiovascular and cerebral systems. However, due to its wide expression in numerous cell types, it is not a plausible HSC gene-based remedial target [21].

In a study by Allers, et al., it was emphasized that CXCR4 surface expression on peripheral and mucosal CD4+ T-cells is not disabled in the CCR $5 \Delta 32 / \Delta 32$ stem cell transplant (SCT) patient. They additionally discovered that CD4+ cells, once altered at a genetic level, had a half-life of about a year and terminated at a slower rate in contrast with the genetically unmodified CD4+ cells when the ART treatment ceased [8].

\section{Engineering stem cell transplantation}

\section{Through Host Cellular Genes Modulation}

As part of strategical approaches to hinder the process of HIV replication at different levels, HSCs are transformed to make them HIV-resistant (Figure 2) [22]. These methods can be categorized into three main strategies: the foremost earmarks the cellular genes required for viral replication, and the focal point of this article will be the newest advances targeting the CCR5 co-receptor which is a requisite for viral entry; the second one targets the HIV gene expression itself; and the last one, using the restriction factors of the host and fusion inhibitors, introduces genes that interfere with HIV replication.

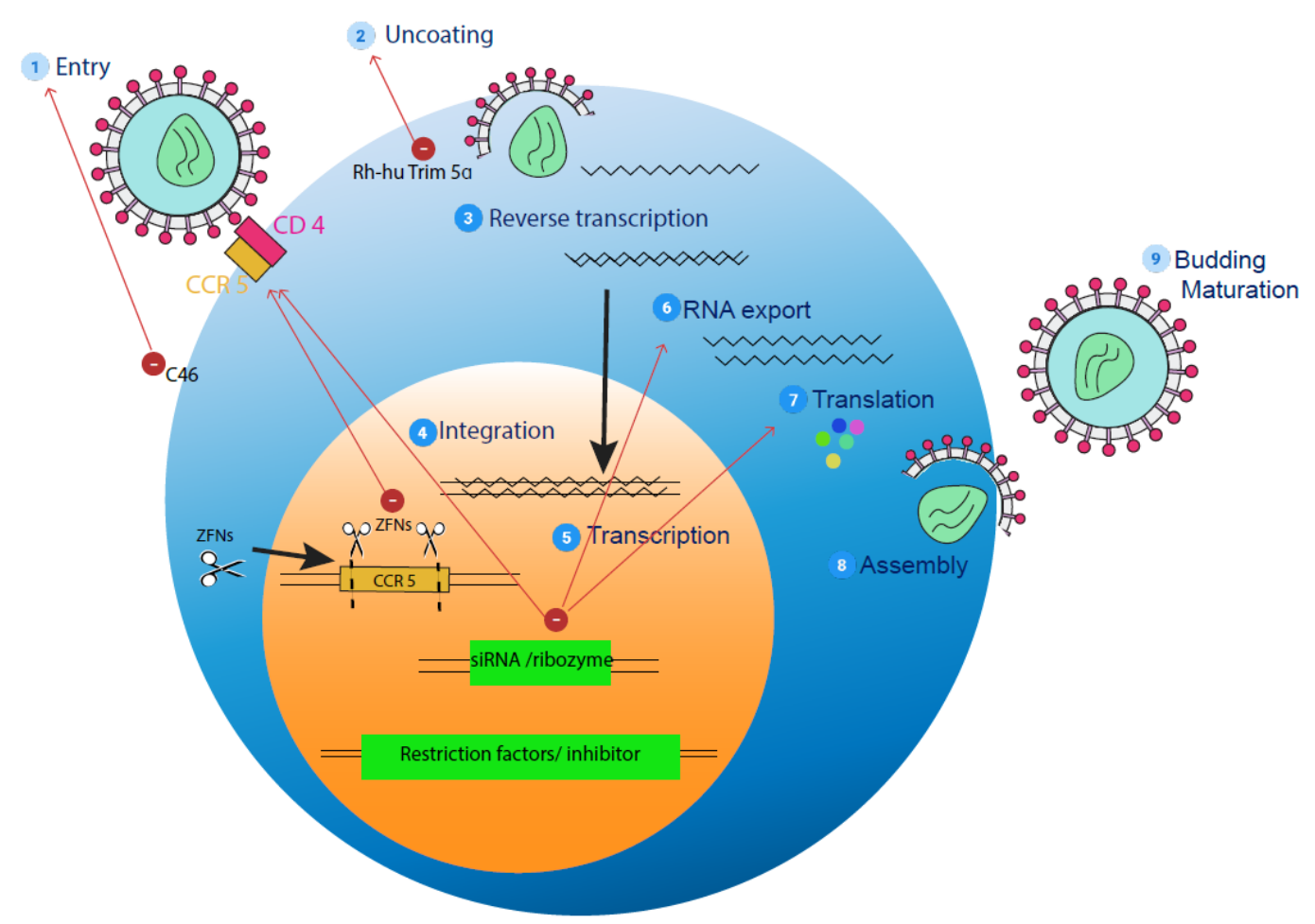

\section{FIGURE 2: HIV life cycle and strategies to engineer HIV- resistant cells}

(1) Entry: HIV enters the host cell by attaching envelope glycoproteins, gp 120 and gp 41 to CD4, utilizing CCR5 or CXCR4 as a co-receptor. Fusion into the cell membrane is inhibited by the membrane-bound form of the T20 inhibitor, the peptide C46. Small interfering RNAs or siRNAs and zinc finger nucleases (ZFNs) can be designed to inhibit entry. 
(2) Uncoating: HIV particle uncoats and releases its RNA into the cell. Rh-hu Trim 5 alpha can help by inhibiting uncoating.

(3) Subsequently, the RNA undergoes reverse transcription.

(4) It now gets incorporated into the genome by integration.

(5) The RNA is transcribed and exported out of the nucleus (6).

(7) It is further translated into protein, which occurs in the cytoplasm of the cell. Short interfering RNA (siRNA) can inhibit RNA export and translation.

(8) The translated viral particle undergoes assembly and leaves the cell to transfect multiple more cells.

Adapted from Zhen A and Kitchen S [22].

The phenomenal case of the "Berlin Patient", the first patient who was declared cured of an HIV infection, further emphasized the prospects of targeting CCR 5 as a potential treatment for HIV infection. This patient had received a bone marrow transplant for acute myeloid leukemia from a donor homozygous for CCR5 $\Delta 32 / \Delta 32$. [8, 23]. HIV was undetectable in the patient for years even consequent to discontinuation of combination antiretroviral therapy. This was attributed to the engrafted donor cells that are believed to have imparted long-term control of the HIV infection post-transplantation.

Despite the success in the aforementioned case, this approach is unsuitable as a mainstay treatment strategy. The rare odds of finding a human leukocyte antigen-matched, CCR5 $\Delta 32 / \Delta 32$ homozygous donor (especially within the non-European populations), and the substantial morbidity and mortality risk attributable to allogeneic stem cell transplantation, have significantly restrained this strategy, to be used only as a last resort for patients with AIDS-associated malignancies.

Gene therapies that modify autologous peripheral blood T-cells or HSCs can be used to mimic the CCR5 $\Delta 32 / \Delta 32$ phenotype seen in the Berlin Patient. In particular, genetically modified HSCs can be used to engineer HIV-1 resistant immunity that would prevent ongoing viral infection. To that end, several gene therapy approaches are being tested to reduce CCR 5 expression or disrupt the CCR5 gene. These include using ribonucleic acid interference (RNAi) [24], using ribozymes to reduce CCR5 RNA levels [25], or using intrabodies [26] and intrakines [27] to target the CCR5 protein directly. RNA interference can be achieved through the stable expression of CCR5 short hairpin RNA (shRNA) from lentiviruses.

A recent study by Shimizu, et al. demonstrated that the transduction of human CD34+ hematopoietic cells with a CCR5-specific shRNA expressing lentivirus appeared to have no major adverse effect on T-cell development in humanized bone marrow/liver/thymus (BLT) mice. Down regulation of CCR5 was found in both human T-cells and monocytes/macrophages in systemic lymphoid tissues, and CCR5 tropic HIV-1 replication was effectively inhibited ex vivo [28].

To directly impair the genomic sequence of CCR5 and subsequently block its expression is another possible approach. To dismiss CCR5 in autologous CD4 T cells, Bobis-Wozowicz et al., in their study, used zinc-finger nuclease (ZFN) with the help of an adenoviral vector. These ZFNs are actually artificial restriction enzymes that can be programmed to cleave 
deoxyribonucleic acid (DNA) at specific sites. Since these breaks naturally induce mutations during their repair, the disruption of the ability of targeted allele, in this case CCR5, to make a functional protein, becomes more plausible. Importantly, only transient ZFN expression is required to permanently disrupt the target genes, and thus it confers very little risk of immune elimination because of antigen presentation [29].

About 50\% of CCR5 alleles in a pool of primary T-cells were identified as being permanently disrupted by transient ZFN expression in the initial studies done by Perez, et al. [30]. One subsequent study concluded that the CCR5 gene was disrupted in 17\% of human CD34+ hematopoietic cells by ZFN via nucleofection using the humanized mouse model, and the modified cells were successfully engrafted in non-obese diabetic (NOD)/severe combined immunodeficiency (SCID)/IL2rynull (NSG) mice. Successful resistance to R5-tropic HIV infection was conferred to cells with this modification. Evidently, rapid selection for CCR5-/cells during HIV infection occurred in the mice transplanted with ZFN modified cells, and these mice had significantly lower HIV-1 levels [31].

These positive outcomes encouraged several clinical trials of ZFN targeting CCR5 in peripheral T-cells (clinicaltrials.gov: NCR00842634, NCT01044654). According to a recent study, ZFN nuclease when delivered by a recombinant adenoviral vector effectively disrupted $>25 \%$ CCR 5 gene in protein kinase $\mathrm{C}$ (PKC) activator pretreated HSCs isolated from granulocyte colonystimulating factor (CSF)-mobilized adults, and these cells underwent multilineage differentiation both in vitro and in vivo [32].

\section{Through HIV Gene Modulation}

HIV genes that are essential for viral replication can also serve as targets for HIV gene therapy. Among them, tat and rev are both transactivators and are critical for HIV-1 infectivity. In stem cells, HIV tat and its overlapping genes have been targeted using hammerhead ribozymes, which are small catalytic RNA molecules engineered to target specific RNA species. A Phase 2 gene therapy trial showed that patients who received tat-vpr-specific ribozyme modified autologous CD34+ cells have higher CD4+ T-cell counts compared to the placebo group, but they have no statistically significant differences in viral load [33].

RNA decoys have also been used to inhibit tat recognition of HIV trans-activating response region (TAR) and inhibit viral replication in vitro [34]. HIV rev, the viral regulatory protein that allows the nucleus to do cytoplasmic transport of unspliced viral RNA, can also be targeted in gene therapy. Dominant mutants or trans-dominant forms of rev have been used to inhibit HIV replication in gene-modified cells [5-6, 34-36]. Decoy rev responsive elements (RREs) have also been tested as potential therapeutic targets and were shown to sequester rev in a clinical trial $[9-10,37]$.

Based on the pattern of HIV gene expression, different stages of the viral replication cycle can also be targeted by RNA interference (RNAi). All viral transcripts, including those encoding tat, rev, gag, pol, nef, vif, env, vpr, as well as long terminal repeats (LTR), are susceptible to RNAi down regulation in cell lines $[15,38]$. A substantial problem of clinical application of RNAi is the virus's high mutation rate and its ability to escape targeted therapy [7,39]. Therefore, as described previously, targeting cellular factors such as CCR 5 by RNAi may be a more attractive gene therapy strategy than targeting viral transcripts.

\section{Through Use of Modified T-Cell Cloned Receptor (TCR)}

Apart from designing HIV-resistant cells, immunity too plays a pivotal role in containing HIV infection. One of the strategies is to genetically modify peripheral blood cells with a 
molecularly cloned T-cell receptor (TCR) or a chimeric molecule to boost the host's antiviral immunity that can redirect the cells to aim at HIV antigens.

The peripheral cells of the patient can be modified by peptide-specific TCR from cloned reactive T-cells [8, 40-42]. In one study, a TCR from a patient that had a sustained and robust CTL response against HIV gag SL9 peptide was molecularly cloned. When genetically modified, CD8 cells were introduced to the primary CD8 cells via transfection; they showed enhanced and polyfunctional immune responses against the viral antigen with an increased capacity to control HIV infection [42].

Numerous hurdles that were encountered in previous studies and trials can be circumvented by stem cell-based anti-HIV immune therapy. The stem cell-based "redirection" approach also has some advantages over previous strategies. In this approach, proper thymic selection of the modified cells and exclusion of endogenous TCR surface expression will be allowed by molecularly cloned TCR, eliminating the chances of generating self-reactive TCR through mispairing [43].

Also, this therapy would allow a long-lived and renewable immunity system to continuously produce antiviral cells that could ultimately lead to HIV eradication. Recently, a study concluded that viral replication was significantly lowered after humanized mice were transplanted with human HSCs genetically modified with an anti-HIV SL9 TCR in comparison with control mice after HIV exposure [44].

The genetic modification of HSCs with a TCR entitle the cells to differentiate in vivo and the stem cells modified by TCR differentiate in to mature CD8+ cells in various tissues [44]. This study highlighted the potential capability of redirecting anti-HIV immunity using HSC recent advances in identifying antigen-specific TCR shall make it possible to molecularly clone multiple TCR from each patient quickly [45].

The cloned TCR, which matches the patient's human leukocyte antigen (HLA) type and viral genome, could be used to genetically modify patient's HSCs which can subsequently allow the development of evolved and sustained modified CD8+ T-cells capable of targeting and eradicating HIV-infected cells.

\section{Potential risks and adverse effects}

It has already been observed that CCR $5 \Delta 32$ mutation protects against HIV, but may also play a negative role in post-infection inflammatory processes, which can not only injure tissue but can also create further pathology [46]. Homozygous patients for CCR $5 \Delta 32$ were found to be at higher risk for a neuroinvasive form of tick-borne encephalitis [47]. In addition, functional CCR5 shall be required to prevent symptoms after infection with West Nile virus (WNV). CCR5 $\Delta 32$ was also associated with the development of early symptoms and more prominent clinical manifestations after infection with WNV [48]. In a study done in mice, the viral load in the central nervous system and mortality increased after an infection with WNV [49]. However, with further enhancement of technology related to the modulation of stem cells, it is expected that the adverse effects and potential risk will diminish [50].

\section{Conclusions}

With the latest technology available, the gene-modifying system has been improved significantly and has now become an appealing strategy for HIV treatment. Of late, HSC-based anti-HIV gene therapy seems promising. This treatment can definitely give long-lasting and renewable immune cells that are either HIV resistant or insusceptible to HIV entry. With further research and with more funds for carrying out this research, HSC-based gene-modification 
therapy can assuredly acquire great results and, may be, can also lead to a cure followed by the eradication of HIV.

\section{Additional Information \\ Disclosures}

Conflicts of interest: In compliance with the ICMJE uniform disclosure form, all authors declare the following: Payment/services info: All authors have declared that no financial support was received from any organization for the submitted work. Financial relationships: All authors have declared that they have no financial relationships at present or within the previous three years with any organizations that might have an interest in the submitted work. Other relationships: All authors have declared that there are no other relationships or activities that could appear to have influenced the submitted work.

\section{Acknowledgements}

We would like to thank Dr. Lakshita Gupta for helping us in improving this article.

\section{References}

1. AIDS by the numbers . (2016). Accessed: 7th October, 2016: http://www.unaids.org/en/resources/documents/2016/AIDS-by-the-numbers.

2. Jha PK, Narayan P, Nair S, Ganju D, Sahu D, Pandey A: An assessment of comprehensive knowledge of HIV/AIDS among slum and non-slum populations in Delhi, India. Open J Prev Med. 2015, 5:259-68. 10.4236/ojpm.2015.56029

3. Mehta V, Mehta S: Assessment of HIV knowledge and awareness in adults of a slum area of Mumbai, India: a cross-sectional study. Int J Community Med Public Health. 2016, 3:314-18. 10.18203/2394-6040.ijcmph20151583

4. Yukl SA, Shergill A, McQuaid K, et al.: Effect of raltegravir-containing intensification on HIV burden and T-cell activation in multiple gut sites of HIV-positive adults on suppressive antiretroviral therapy. Aids. 2010, 24:2451-2460. 10.1097/QAD.0b013e32833ef7bb

5. Deeks SG: HIV Infection, inflammation, immunosenescence, and aging medicine. Annu Rev Med. 2011, 62:141-155. 10.1146/annurev-med-042909-093756

6. Volberding PA, Deeks SG: Antiretroviral therapy and management of HIV infection. Lancet. 2010, 376:49-62. 10.1016/S0140-6736(10)60676-9

7. Liu R, Paxton WA, Choe S, et al.: Homozygous defect in HIV-1 coreceptor accounts for resistance of some multiply-exposed individuals to HIV-1 infection. Cell. 1996, 86:367-377. 10.1016/S0092-8674(00)80110-5

8. Weiss RA: Thirty years on: HIV receptor gymnastics and the prevention of infection . BMC Biology. 2013, $11:$ e57. 10.1186/1741-7007-11-57

9. Yu M, Poeschla E, Wong-Staal F: Progress towards gene therapy for HIV infection. Gene Ther. 1194, 1:13-26.

10. Piche A: Gene therapy for HIV infections: Intracellular immunization. Can J Infect Dis. 1999, 10:307-312. 10.1155/1999/914379

11. Mehandru S, Poles MA, Tenner-Racz K, et al.: Primary HIV-1 infection is associated with preferential depletion of CD4+ T lymphocytes from effector sites in the gastrointestinal tract. J Exp Med. 2004, 200:761-70. 10.1084/jem.20041196

12. Schneider T, Jahn HU, Schmidt W, Riecken EO, Zeitz M, Ullrich R: Loss of CD4 T lymphocytes in patients infected with human immunodeficiency virus type 1 is more pronounced in the duodenal mucosa than in the peripheral blood. Berlin Diarrhea/Wasting Syndrome Study Group . Gut. 1995, 37:524-529. 10.1136/gut.37.4.524

13. Veazey RS, DeMaria M, Chalifoux LV, et al.: Gastrointestinal tract as a major site of CD4+ T cell depletion and viral replication in SIV infection. Science. 1998, 280:427-431.

10.1126/science.280.5362.427

14. HIV attachment process. US National Institutes of Health - National Institute of Allergy and Infectious Diseases. (2007). Accessed: October 16th, 2016: 
https://commons.wikimedia.org/wiki/File\%3AHIV_attachment.gif.

15. Allers K, Hütter G, Hofmann J, et al.: Evidence for the cure of HIV infection by CCR $5 \Delta 32 / \Delta 32$ stem cell transplantation. Blood. 2011, 117:2791-2799. 10.1182/blood-2010-09-309591

16. Berger EA, Murphy PM, Farber JM: Chemokine receptors as HIV-1 coreceptors: roles in viral entry, tropism, and disease. Annu Rev Immunol. 1999, 17:657-700.

10.1146/annurev.immunol.17.1.657

17. Carrington M, Kissner T, Gerrard B, Ivanov S, O'Brien SJ, Dean M: Novel alleles of the chemokine-receptor gene CCR5. Am J Hum Genet. 1997, 61:1261-1267. 10.1086/301645

18. de Roda Husman AM, Koot M, Cornelissen M, et al.: Association between CCR5 genotype and the clinical course of HIV-1 infection. Ann Intern Med. 1997, 127:882-890. 10.7326/00034819-127-10-199711150-00004

19. Stephens JC, Reich DE, Goldstein DB, et al.: Dating the origin of the CCR5- $\triangle 32$ AIDSresistance allele by the coalescence of haplotypes. Am J Hum Genet. 1998, 62(6):1507-1515. 10.1086/301867

20. Gilliam BL, Riedel DJ, Redfield RR: Clinical use of CCR5 inhibitors in HIV and beyond . J Transl Med. 2011, 9:S9. 10.1186/1479-5876-9-S1-S9

21. Kiem HP, Jerome KR, Deeks SG, McCune JM: Hematopoietic stem cell-based gene therapy for HIV disease. Cell Stem Cell. 2012, 10:137-47. 10.1016/j.stem.2011.12.015

22. Zhen A, Kitchen S: Stem-cell-based gene therapy for HIV infection. Viruses. 2014, 6:1-12. 10.3390/v6010001

23. Hütter G, Nowak D, Mossner M, Ganepola S, Müßig A, Allers K, Schneider T, Hofmann J, Kücherer C, Blau O, Blau IW: Long-term control of HIV by CCR5 Delta32/Delta32 stem-cell transplantation. N Engl J Med. 2009, 360:692-698. 10.1056/NEJMoa0802905

24. Qin XF, An DS, Chen IS, Baltimore D: Inhibiting HIV-1 infection in human T cells by lentiviral-mediated delivery of small interfering RNA against CCR5. Proc Natl Acad Sci USA. 2003, 100:183-188. 10.1073/pnas.232688199

25. Bai J, Gorantla S, Banda N, Cagnon L, Rossi J, Akkina R: Characterization of anti-CCR5 ribozyme-transduced CD34+ hematopoietic progenitor cells in vitro and in a SCID-hu mouse model in vivo. Mol Ther. 2000, 1:244-254. 10.1006/mthe.2000.0038

26. Swan CH, Bühler B, Tschan MP, Barbas C, Torbett BE: T-cell protection and enrichment through lentiviral CCR5 intrabody gene delivery. Gene Ther. 2006, 13:1480-1492. 10.1038/sj.gt.3302801

27. Schroers R, Davis CM, Wagner HJ, Chen SY: Lentiviral transduction of human T-lymphocytes with a RANTES intrakine inhibits human immunodeficiency virus type 1 infection. Gene Ther. 2002, 9:889-897. 10.1038/sj.gt.3301711

28. Shimizu S, Hong P, Arumugam B: A highly efficient short hairpin RNA potently downregulates CCR5 expression in systemic lymphoid organs in the hu-BLT mouse model. Blood. 2010, 115:1534-1544. 10.1182/blood-2009-04-215855

29. Bobis-Wozowicz S, Osiak A, Rahman SH, Cathomen T: Targeted genome editing in pluripotent stem cells using zinc-finger nucleases. Methods. 2011, 53:339-346. 10.1016/j.ymeth.2010.12.019

30. Perez EE, Wang J, Miller JC, Jouvenot Y, et al.: Establishment of HIV-1 resistance in CD4+ T cells by genome editing using zinc-finger nucleases. Nature Biotechnol. 2008, 26:808-816. 10.1038/nbt1410

31. Holt N, Wang J, Kim K, et al.: Human hematopoietic stem/progenitor cells modified by zincfinger nucleases targeted to CCR5 control HIV-1 in vivo. Nature Biotechnol. 2010, 28:839847. 10.1038/nbt.1663

32. Li L, Krymskaya L, Wang J, et al.: Genomic editing of the HIV-1 coreceptor CCR5 in adult hematopoietic stem and progenitor cells using zinc finger nucleases. Mol Ther. 2013, 21:1259-1269. 10.1038/mt.2013.65

33. Mitsuyasu RT, Merigan TC, Carr A, et al.: Phase 2 gene therapy trial of an anti-HIV ribozyme in autologous CD34+ cells. Nature Med. 2009, 15(3):285-292. 10.1038/nm.1932

34. Kang EM, de Witte M, Malech H, et al.: Nonmyeloablative conditioning followed by transplantation of genetically modified HLA-matched peripheral blood progenitor cells for hematologic malignancies in patients with acquired immunodeficiency syndrome. Blood. 2002, 99(2):698-701. 10.1182/blood.V99.2.698

35. Bonyhadi ML, Moss K, Voytovich A, et al.: RevM10-expressing T cells derived in vivo from transduced human hematopoietic stem-progenitor cells inhibit human immunodeficiency 
virus replication. J Virol. 1997, 71:4707-4716.

36. Podsakoff GM, Engel BC, Carbonaro DA, Choi C, et al.: Selective survival of peripheral blood lymphocytes in children with HIV-1 following delivery of an anti-HIV gene to bone marrow CD34+ cells. Mol Ther. 2005, 12:77-86. 10.1016/j.ymthe.2005.02.024

37. Kohn DB, Bauer G, Rice CR, Rothschild JC, Carbonaro DA, Valdez P, Hao QL, Zhou C, Bahner I, Kearns K, Brody K: A clinical trial of retroviral-mediated transfer of arev-responsive element decoy gene into CD34+ cells from the bone marrow of human immunodeficiency virus-1infected children. Blood. 1999, 94:368-371.

38. Jacque JM, Triques K, Stevenson: Modulation of HIV-1 replication by RNA interference . Nature. 2002, 418(6896):435-438. 10.1038/nature00896

39. Das AT, Brummelkamp TR, Westerhout EM, et al.: Human immunodeficiency virus type 1 escapes from RNA interference-mediated inhibition. J Virol. 2004, 78:2601-2605.

10.1128/JVI.78.5.2601-2605.2004

40. Joseph A, Zheng JH, Chen K, et al.: Inhibition of in vivo HIV infection in humanized mice by gene therapy of human hematopoietic stem cells with a lentiviral vector encoding a broadly neutralizing anti-HIV antibody. J Virol. 2010, 84:6645-6653. 10.1128/JVI.02339-09

41. Cooper LJ, Kalos M, Lewinsohn DA, Riddell SR, Greenberg PD: Transfer of specificity for human immunodeficiency virus type 1 into primary human $\mathrm{T}$ lymphocytes by introduction of T-cell receptor genes. J Virol. 2000, 74:8207-8212. 10.1128/JVI.74.17.8207-8212.2000

42. Varela-Rohena A, Molloy PE, Dunn SM, et al.: Control of HIV-1 immune escape by CD8 T cells expressing enhanced T-cell receptor. Nature Med. 2008, 14:1390-5. 10.1038/nm.1779

43. Vatakis DN, Arumugam B, Kim SG, et al.: Introduction of exogenous T-cell receptors into human hematopoietic progenitors results in exclusion of endogenous T-cell receptor expression. Mol Ther. 2013, 21:1055-1063. 10.1038/mt.2013.28

44. Kitchen SG, Levin BR, Bristol G, Rezek V, Kim S, Aguilera-Sandoval C, Balamurugan A, Yang OO, Zack JA: In vivo suppression of HIV by antigen specific T cells derived from engineered hematopoietic stem cells. PLOS Pathog. 2012, 8:e1002649. 10.1371/journal.ppat.1002649

45. Balamurugan A, Ng HL, Yang OO: Rapid T cell receptor delineation reveals clonal expansion limitation of the magnitude of the HIV-1-specific CD8+ T cell response. J Immunol. 2010, 185:5935-5942. 10.4049/jimmunol.1002236

46. Klein RS: A moving target: the multiple roles of CCR5 in infectious diseases . J Infect Dis. 2008, 197:183-186. 10.1086/524692

47. Kindberg E, Mickienè A, Ax C, Åkerlind B, Vene S, Lindquist L, Lundkvist Å, Svensson L: A deletion in the chemokine receptor 5 (CCR5) gene is associated with tickborne encephalitis. J Infect Dis. 2008, 197:266-269. 10.1086/524709

48. Lim JK, Louie CY, Glaser C, et al.: Genetic deficiency of chemokine receptor CCR5 is a strong risk factor for symptomatic West Nile virus infection: a meta-analysis of 4 cohorts in the US epidemic. J Infect Dis. 2008, 197:262-265. 10.1086/524691

49. Glass WG, Lim JK, Cholera R, Pletnev AG, Gao JL, Murphy PM: Chemokine receptor CCR5 promotes leukocyte trafficking to the brain and survival in West Nile virus infection. J Exp Med. 2005, 202:1087-98. 10.1084/jem.20042530

50. Aiuti A, Cattaneo F, Galimberti S, et al.: Gene therapy for immunodeficiency due to adenosine deaminase deficiency. N Engl J Med. 2009, 360:447-458. 10.1056/NEJMoa0805817 